DOI: 10.20472/IAC.2018.044.035

KEITA NISHIO

Aoyama Gakuin University, Japan

TAKASHI KABURAGI

Aoyama Gakuin University, Japan

SATOSHI KUMAGAI

Aoyama Gakuin University, Japan

TOSHIYUKI MATSUMOTO

Aoyama Gakuin University, Japan

YOSUKE KURIHARA

Aoyama Gakuin University, Japan

\title{
UNRESTRICTIVE DECUBITUS ESTIMATION SYSTEM WITH HIGH-SENSITIVITY PRESSURE SENSOR
}

\begin{abstract}
:
Estimation decubitus during sleep is essential to prevent the bedsore. However, utilizing wrist-worn device to monitor the decubitus during sleep required to attach the device for whole night. Hence, this research proposes an unrestrictive decubitus estimation system with high-sensitivity pressure sensors. In the proposed system, a high-sensitivity pressure sensor is placed under a bed mattress. The signal from the pressure sensor is processed by a three band pass filters (BFPs) with cut off frequencies $0.16-0.8 \mathrm{~Hz}, 0.8-16 \mathrm{~Hz}$ and $0.16-16 \mathrm{~Hz}$ to measure respiration, pulse, and body movement respectively. In the case of prone position, since the heart is close to the sensor, peak frequency of pulse signal is low and the intensity is large. In the case of lateral position, the pressure sensor could not detect respiration because the movement of the diaphragm does not transfer to the mattress directly. Hence, the amplitude of respiration signal becomes small. In a supine position, both respiration and pulse signal are measured normally. Furthermore, since the movement signal is affected by the turning over, we do not apply the signal containing the turning over to the estimation of the decubitus. Based on above relationship between characteristics of these bio-signals and decubitus, we estimate each decubitus. In order to evaluate the validity of the proposed system, we carried out an experiment with five subjects. As the result, accuracy of the proposed method was at least $80 \%$.
\end{abstract}

\section{Keywords:}

bedsore, high-sensitivity pressure sensor, decubitus estimation, unrestrictive bio-signal sensing

JEL Classification: 110 